\title{
Effect of Sainfoin (Onobrychis viciifolia) Pellets on Rumen Microbiome and Histopathology in Lambs Exposed to Gastrointestinal Nematodes
}

\author{
Daniel Petrič ${ }^{1,2}$, Michaela Komáromyová ${ }^{2,3}$, Dominika Batt ányi ${ }^{1} \mathbb{D}$, Martyna Kozłowska ${ }^{4,5}$, Weronika Filipiak ${ }^{4}$, \\ Anna Lukomska ${ }^{6}{ }^{(D)}$, Sylwester Ślusarczyk ${ }^{7}$ (D), Malgorzata Szumacher-Strabel ${ }^{4}$, Adam Cieślak ${ }^{4}$ (D), \\ Marián Várady ${ }^{3}\left(\mathbb{D}\right.$, Svetlana Kišidayová ${ }^{1}(\mathbb{D})$ and Zora Váradyová ${ }^{1, *(\mathbb{D})}$
}

Citation: Petrič, D.; Komáromyová, M.; Batt'ányi, D.; Kozłowska, M.; Filipiak, W.; Łukomska, A.;

Ślusarczyk, S.; Szumacher-Strabel, M.; Cieślak, A.; Várady, M.; et al. Effect of Sainfoin (Onobrychis viciifolia) Pellets on Rumen Microbiome and

Histopathology in Lambs Exposed to Gastrointestinal Nematodes. Agriculture 2022, 12, 301. https:/ / doi.org/10.3390/agriculture12020301

Academic Editor: Robert Dixon

Received: 11 January 2022

Accepted: 16 February 2022

Published: 21 February 2022

Publisher's Note: MDPI stays neutral with regard to jurisdictional claims in published maps and institutional affiliations.

Copyright: (C) 2022 by the authors. Licensee MDPI, Basel, Switzerland. This article is an open access article distributed under the terms and conditions of the Creative Commons Attribution (CC BY) license (https:// creativecommons.org/licenses/by/ $4.0 /)$.
1 Centre of Biosciences of Slovak Academy of Sciences, Institute of Animal Physiology, Šoltésovej 4-6, 04001 Košice, Slovakia; petric@saske.sk (D.P.); mravcakova@saske.sk (D.B.); kisiday@saske.sk (S.K.)

2 University of Veterinary Medicine and Pharmacy in Košice, Komenského 73, 04181 Košice, Slovakia; komaromyova@saske.sk

3 Institute of Parasitology, Slovak Academy of Sciences, Hlinkova 3, 04001 Košice, Slovakia; varady@saske.sk

4 Department of Animal Nutrition, Poznan University of Life Sciences, Wołyńska 33, 60-637 Poznan, Poland; mkozowska73@gmail.com (M.K.); weronika.filipiak@up.poznan.pl (W.F.); malgorzata.szumacher@up.poznan.pl (M.S.-S.); adam.cieslak@up.poznan.pl (A.C.)

5 Institute of Genetics and Animal Biotechnology Polish Academy of Sciences, Postepu 36A, 05-552 Magdalenka, Poland

6 Department of Preclinical Sciences and Infectious Diseases, Poznan University of Life Sciences, Wołyńska 35, 60-637 Poznan, Poland; anna.lukomska@up.poznan.pl

7 Department of Pharmaceutical Biology and Botany, Wroclaw Medical University, Borowska 211, 50-556 Wroclaw, Poland; sylwester.slusarczyk@umed.wroc.pl

* Correspondence: varadyz@saske.sk; Tel.: +421-55-792-2972

\begin{abstract}
Our study analyzed the ruminal fermentation and microbiome, hematological profile, and abomasal histopathology of lambs experimentally infected with a gastrointestinal nematode (GIN) and fed sainfoin pellets (SFPs; $600 \mathrm{~g}$ DM/d/animal) for $14 \mathrm{~d}$. Twenty-four lambs infected with Haemonchus contortus were divided into two separated groups: animals fed meadow hay (control) and animals fed SFPs. The ruminal contents, fermentation parameters, and microbiome in vitro and in vivo were determined using molecular and microscopic techniques. Ruminal contents in the SFP group indicated smaller populations of Archaea $(p<0.001)$, Methanomicrobiales $(p=0.009)$, and lower methane concentrations in vitro $(p=0.046)$ and in vivo $(p=0.030)$ than the control group. The relative abundance of Butyrivibrio fibrisolvens quantified by real-time PCR was higher in the lambs with the SFP diet $(p=0.05)$. Haemonchosis affected the number of red blood cells of the lambs $(p<0.001)$. The lambs in the SFP group had a higher percentage of damaged abomasa glands than did the control group ( $p=0.004$ ). The consumption of SFPs by GIN-infected lambs may affect ruminal methanogens and subsequently decrease methane emission without undesirable changes in the ruminal microbiome or the health of the animals.
\end{abstract}

Keywords: bacteria; flavonoids; hematological profiles; methane concentration; plant bioactive components; ruminal fermentation; sheep

\section{Introduction}

Parasitic infection in ruminants, mainly by gastrointestinal nematodes (GINs), influences the intensity of emissions of greenhouse gases and substantially increases the yield of methane emission compared to uninfected animals [1,2]. Promising new nutraceuticals containing bioactive components in ruminant nutrition, however, could have both anthelmintic and anti-methanogenic properties [3,4]. Plant additives with bioactive components can modulate the bacterial, archaeal, and eukaryotic populations in the rumen by interactions 
between diet and the microbes because the microbiome plays a crucial functional role in nitrogen use, fermentation, and methane concentration [5].

Plant bioactive components such as flavonoids and condensed tannins (CTs) in feeds have the potential to reduce environmental methane pollution from ruminants by complex bioactivity occurring simultaneously in plants and animals [6]. The main bioactive components in the tanniferous legume sainfoin (Onobrychis viciifolia) are flavonoids and CTs formed by the polymerization of flavan-3-ols, with high proportions of prodelphinidins $(70 \%)$ and procyanidins (30\%) [7]. Tanniferous forages are rich in prodelphinidins, have higher antiparasitic activity, and have the effect of reducing methane emissions [8-10]. Sainfoin also reduces the degradation of feed proteins without affecting the digestibility of the nonprotein fraction, thereby increasing the flow of non-ammonia nitrogen and essential amino acids into the small intestine and reducing urinary nitrogen losses [11,12]. The ability of sainfoin to reversibly bind proteins leads to a reduction in GIN parasitism in small ruminants [13,14]. Many studies have focused on the use of sainfoin for its nutritional and anthelmintic effects, but it also contains beneficial flavonoids with similar mechanisms of action as tannins and similarly interferes with the biology of GINs [15]. Combining CTs with quercetin or luteolin identified synergistic anthelmintic effects between tannins and flavonoid monomers [16]. The production of sainfoin pellets (SFPs) at high temperatures and pressure does not affect their bioactivities associated with antioxidative properties [17]. Based on previous studies [18,19], we hypothesized that SFPs would also contribute to desired changes in the ruminal microbiome and histopathology in lambs loaded with parasites.

Analyses of ruminal microbiomes and histopathological observations are needed to identify the possible consequences of bioactive components used in the nutrition of parasiteladen lambs. Our aim was to (1) identify the main flavonoids and phenolic compounds of the SFPs and (2) determine the ruminal fermentation and microbiome, hematological profile, and histopathology of the abomasum of lambs infected with GINs during consumption of SFPs for $14 \mathrm{~d}$.

\section{Materials and Methods}

\subsection{Ethics Statement}

This study was conducted following the guidelines of the Declaration of Helsinki and national legislation in the Slovak Republic (G.R. 377/2012; Law 39/2007) for the care and use of research animals. The experimental protocol was approved by the Ethical Committee of the Institute of Parasitology of the Slovak Academy of Sciences on 22 November 2020 (protocol code 2020/21).

\subsection{Animals, Diets, and Experimental Design}

We housed 24 male lambs (Improved Valachian) 3-4 months of age with an average initial body weight of $15.0 \pm 2.07 \mathrm{~kg}$ in common stalls for $7 \mathrm{~d}$ for a period of adaptation and another $7 \mathrm{~d}$ for acclimatizing to feeding, with free access to water. The lambs were obtained from a commercial farm (PD Ružín-Ružín farm, Kysak, Slovakia) where they were also housed during the experiment. Each animal was fed daily meadow hay $(\mathrm{MH})$ ad libitum and $300 \mathrm{~g}$ dry matter (DM) Mikrop ČOJ, a commercial concentrate (MIKROP, Čebín, Czech Republic). The number of animals used in the experiment was assigned following VICH GL13 guidelines (Veterinary International Committee on Harmonization-Efficacy of anthelmintics: specific requirements for ovine). At the beginning of the experiment-day (D) 0, all parasite-free lambs were infected orally with approximately 5000 third-stage larvae of the MHCo1 strain of Haemonchus contortus susceptible to anthelmintics [20]. A modified McMaster technique [21] with a sensitivity of 50 eggs per gram (EPG) of faeces was used for detecting H. contortus eggs on D30. The lambs were divided into two groups of twelve animals each (one stall per group) on D30 after infection, when all parasites had matured to the adult stage: control animals fed $\mathrm{MH}$ (control, $\mathrm{MH}, 600 \mathrm{~g} \mathrm{DM} / \mathrm{d} / \mathrm{animal}$ ) and animals fed sainfoin pellets (SFPs, $600 \mathrm{~g} \mathrm{DM} / \mathrm{d} / \mathrm{animal}$ ). Both groups continued to be fed commercial concentrate ( $300 \mathrm{~g} \mathrm{DM} / \mathrm{d} /$ animal). All lambs were positive with a mean 
EPG of $9405 \pm 4584$ in the SFPs group and a mean of $11420 \pm 372$ in the control group. SFPs were obtained from a commercial source (NATURE'S BEST, EQUOVIS GmbH, Münster, Germany). This feeding scheme continued for $14 \mathrm{~d}$. The lambs were weighed at the end of the experiment and had an average final body weight of $18.3 \pm 3.22 \mathrm{~kg}$. All animals were killed at the end of the experiment following the rules of the European Commission (Council Regulation 1099/2009) for slaughtering procedures [22].

\subsection{Experiment In Vitro}

In vitro gas fermentation technique (IVFT) has been widely used to evaluate the nutritive value of feeds for ruminants and to assess the effect of different nutritional strategies on methane $\left(\mathrm{CH}_{4}\right)$ production. Therefore, IVFT using batch-culture incubations of buffered ruminal fluid incubated at $39^{\circ} \mathrm{C}$ for $24 \mathrm{~h}$ under anaerobic conditions was used [23]. Control animals were donor animals for control groups and SFPs animals were donor animals for SFPs groups for the in vitro experiment. At the end of the experiment the ruminal contents (RCs) were taken from each lamb of each group immediately after slaughter in the abattoir, packed in prewarmed flasks and transported to the laboratory. RCs were pushed through four layers of gauze and pooled in equal volumes based on control and SFP groups. The pooled RCs were purged with $\mathrm{CO}_{2}$, mixed with McDougall's buffer [24] in a 1:2 ratio, and dispensed in volumes of $35 \mathrm{~mL}$ into fermentation bottles $(120 \mathrm{~mL}$ ) containing $250 \mathrm{mg}$ (DM basis) of substrate. The meadow hay or SFPs were used as the substrates of a ration with commercial concentrate $(800: 200, w / w)$ as the components of the diets for the controls and SFP groups for in vitro experiment. Commercial concentrate, $\mathrm{MH}$, and SFPs were ground using a grinder (Molina, MIPAM, České Budějovice, Czech Republic) and sieved through $0.15-0.40 \mathrm{~mm}$ screens. The in vitro experiment had a completely randomized design using the two diets (control and SFP) in fermentations with the two inocula of ruminal fluids (control and SFPs), with three replicates (three incubation bottles) for each diet and inoculum. The in vitro experiment was repeated three times within three consecutive days $(n=3 \times 3)$.

\subsection{Chemical Analysis of the Dietary Substrates}

The chemical compositions of the dietary substrates (Table 1) were analyzed using standard methods $[25,26]$.

Table 1. Chemical compositions of the dietary substrates.

\begin{tabular}{ccccccc}
\hline Substrate & $\begin{array}{c}\text { DM } \\
\text { (g/kg) }\end{array}$ & $\begin{array}{c}\text { NDF } \\
\text { (g/kg DM) }\end{array}$ & $\begin{array}{c}\text { ADF } \\
\text { (g/kg DM) }\end{array}$ & $\begin{array}{c}\text { CP } \\
(\mathbf{g} / \mathbf{k g ~ D M})\end{array}$ & $\begin{array}{c}\mathbf{N} \\
\text { (g//kg DM) }\end{array}$ & $\begin{array}{c}\text { Ash } \\
\text { (g/kg DM) }\end{array}$ \\
\hline Meadow hay & 885 & 640 & 423 & 84 & 14 & 84 \\
Concentrate & 888 & 231 & 130 & 211 & 34 & 104 \\
SFPs & 918 & 460 & 357 & 121 & 19 & 100 \\
\hline
\end{tabular}

DM, dry matter; NDF, neutral detergent fiber; $\mathrm{ADF}$, acidic detergent fiber; $\mathrm{CP}$, crude protein; $\mathrm{N}$, nitrogen; SFPs, sainfoin pellets.

\subsection{Analysis of Bioactive Compounds}

SFPs were ground to a fine powder, and $100 \mathrm{mg}$ were extracted three times with $80 \% \mathrm{MeOH}$ at $40^{\circ} \mathrm{C}$ for $60 \mathrm{~min}$. The extracts were evaporated to dryness and were then dissolved in $2 \mathrm{~mL}$ of Milli-Q water (acidified with $0.2 \%$ formic acid) and purified by solidphase extraction using an Oasis HLB 3 cc Vac Cartridge (Waters Corp., Milford, CT, USA) as was previously described [27]. Bioactive compounds were analyzed by ultrahigh resolution mass spectrometry (UHRMS) on a Dionex UltiMate 3000RS system (Thermo Scientific, Darmstadt, Germany) with a charged aerosol detector connected to a high-resolution quadrupole time-of-flight mass spectrometer (Compact, Bruker Daltonik GmbH, Bremen, Germany). Phenolic acid and flavonoids were identified chromatographically on a Kinetex C18 column $(2.1 \times 100 \mathrm{~mm}, 2.6 \mu \mathrm{m}$, Phenomenex, Torrance, CA, USA), with mobile phase A consisting of $0.1 \%(v / v)$ formic acid in water and mobile phase B consisting of $0.1 \%(v / v)$ formic acid in acetonitrile, as was previously described [19]. Stock solutions of hyperoside and chlorogenic acid were prepared in $80 \% \mathrm{MeOH}$ at concentrations of 2.5 and $3.6 \mathrm{mg} / \mathrm{mL}$, 
respectively, and kept frozen until used. Calibration curves for these two compounds were constructed based on seven concentration points (from 500 to $3.6 \mu \mathrm{g} / \mathrm{mL}$ ). Hyperoside was used to calculate the number of flavonoids identified in the extract, and chlorogenic acid was used for phenolic acids, using Bruker QuantAnalysis 4.3 software (Bruker Daltonik $\mathrm{GmbH}$, Bremen, Germany). All analyses were performed in triplicate.

\subsection{Basic Ruminal Fermentation Analysis}

RC samples from the in vitro and in vivo experiments were collected for determining $\mathrm{pH}$, methane, volatile fatty acids (VFAs), ammonia concentrations, in vitro DM digestibility (IVDMD), and population of ruminal microorganisms (bacteria, protozoa, and methanogens). Concentrations of methane in vitro and VFAs were determined by gas chromatography on a PerkinElmer Clarus 500 gas chromatograph (Perkin Elmer, Inc., Shelton, CT, USA) [28]. Methane concentration in vivo was calculated by measuring the molar proportions of the VFAs in the rumen as: $57.5 \mathrm{~mol}$ glucose $=65 \mathrm{~mol}$ acetate +20 mol propionate +15 mol butyrate $+60 \mathrm{~mol} \mathrm{CO} 2+35 \mathrm{~mol} \mathrm{CH}_{4}+25 \mathrm{~mol} \mathrm{H}_{2} \mathrm{O}$ [29]. The concentration of ammonia-N was determined using the phenol-hypochlorite method [30].

\subsection{Rumen Microbial Quantification}

Samples for counting ciliate protozoa from the RCs were fixed in equal volumes of $8 \%$ formaldehyde, and the protozoa were counted and identified microscopically [31]. Total bacteria, Archaea, Methanobacteriales, and Methanomicrobiales from the in vitro experiment and Archaea and Methanobacteriales from the in vivo experiment were quantified using fluorescence in situ hybridization as described previously [32]. DNA for quantifying bacteria was isolated from the ruminal samples using a Mini Bead-Beater (BioSpec, Bartlesville, OK, USA) to lyse the cells [33] followed by purification using a QIAamp DNA Stool Mini Kit (Qiagen, Hilden, Germany). DNA concentrations and qualities were measured using a NanoPhotometer R NP80 (Implen GmbH, München, Germany). Total bacteria, Streptococcus bovis, Butyrivibrio proteoclasticus, B. fibrisolvens, Fibrobacter succinogenes, Megasphaera elsdenii, Ruminococcus albus, R. flavefaciens, and the genera Prevotella and Lactobacillus were quantified by real-time PCR using the PCR primers [34-39].

\subsection{Hematological Parameters}

Samples of blood were collected from the jugular vein of each animal on D0, D23, D30, D37, and D44. Basic hematological parameters were determined using an Abbott CELL-DYN 3700 hematological analyzer (Global Medical Instrumentation, Inc., Ramsey, NJ, USA).

\subsection{Histopathology}

Samples of fresh abomasal tissues were washed in a phosphate buffer $(0.1 \mathrm{M}, \mathrm{pH} 7.4)$, put in plastic containers, and fixed in a $10 \%$ buffered FA solution as pieces of tissue spread on a flat piece of polystyrene as previously described [19]. The fixed material was processed using a series of reagents in the following sequence: $75 \%$ alcohol for $1 \mathrm{~h}, 90 \%$ alcohol for $1 \mathrm{~h}, 95 \%$ alcohol for $1 \mathrm{~h}, 100 \%$ alcohol 3 times for $1 \mathrm{~h}$. Then, the material was cleared in xylene 3 times for $1 \mathrm{~h}$. The material was infiltrated in paraffin 3 times for $1 \mathrm{~h} 20 \mathrm{~min}$. The described steps took place in a tissue processor (Excelsior AS Thermo Scientific, Runcorn, UK). Afterward, specimens were embedded in Paraplast PLUS paraffin blocks (Leica, Buffalo Grove, IL, USA), which were then cut with a rotary microtome into sections $2.5 \mu \mathrm{m}$ thick. Slides with a paraffin section were automatically stained with hematoxylin and eosin (Varistain Gemini Thermo Scientific, Runcorn, UK). An Axio Lab. A1 microscope (Carl Zeiss, Jena, Germany) equipped with a Zeiss Axiocam ERc 5 s digital camera was used for histological evaluation. Photographs were analyzed and recorded using ZEN 2.3 (blue edition) software (Carl Zeiss Microscopy GmbH, Oberkochen, Germany, 2011). 


\subsection{Statistical Analysis}

Data for the fermentation parameters and microbial populations were analyzed using an unpaired $t$-test (GraphPad Prism 8; GraphPad Software, Inc., San Diego, CA, USA). Twoway analyses of variance were used for analyzing the hematological parameters as models representing the two animal groups (control and SFPs) and sampling days (D0-D44). The effects included in the model were treatment $(\mathrm{T})$, time, and the interaction between them $(\mathrm{T} \times$ time). Results were considered significant at $p<0.05$.

\section{Results}

\subsection{Phytochemicals}

The phytochemical substances in the SFPs consisted of $32.56 \mathrm{~g} / \mathrm{kg}$ DM flavonoids, $4.68 \mathrm{~g} / \mathrm{kg}$ DM phenolics, and $0.37 \mathrm{~g} / \mathrm{kg}$ DM others (glycosides, hydroxy fatty acids, and a derivative of cinnamic acid, Table 2).

Table 2. The main phytochemicals in the $\mathrm{MeOH}$ extract of the sainfoin pellets.

\begin{tabular}{|c|c|c|c|c|c|c|c|c|}
\hline No. & RT (min) & UV (nm) & $\mathbf{m} / \mathbf{z}[\mathbf{M}-\mathrm{H}]^{-}$ & $\begin{array}{l}\text { MS }{ }^{2} \text { Main } \\
\text { Ion }\end{array}$ & $\begin{array}{c}\text { MS }^{2} \\
\text { Fragments }\end{array}$ & Formula & Compound & $\mathrm{mg} / \mathrm{g} \mathrm{DM}$ \\
\hline 1 & 2.4 & 261,296 & 153.018 & 109.0291 & & $\mathrm{C}_{7} \mathrm{H}_{6} \mathrm{O}_{4}$ & 3,5-Dihydroxybenzoic acid & 0.26 \\
\hline 2 & 2.6 & 261,296 & 153.018 & 109.0291 & & $\mathrm{C}_{7} \mathrm{H}_{6} \mathrm{O}_{4}$ & 2,4-Dihydroxybenzoic acid & 0.29 \\
\hline 3 & 4.6 & $245 \mathrm{sh}, 348$ & 175.0601 & 157.0488 & 131,115 & $\mathrm{C}_{7} \mathrm{H}_{12} \mathrm{O}_{5}$ & 3-Isopropylmalic acid & 0.28 \\
\hline 4 & 5.1 & $248 \mathrm{sh}, 326$ & 353.0884 & 191.0557 & & $\mathrm{C}_{16} \mathrm{H}_{18} \mathrm{O}_{9}$ & Trans-5-caffeoylquinic acid & 0.37 \\
\hline 5 & 5.9 & & 367.1034 & 193.0501 & 173.134 & $\mathrm{C}_{17} \mathrm{H}_{20} \mathrm{O}_{9}$ & 3-O-Caffeoyl-4-O-methylquinate & 0.24 \\
\hline 6 & 6.2 & 282,316 & 239.0563 & 179.034 & $195,221,149$ & $\mathrm{C}_{11} \mathrm{H}_{12} \mathrm{O}_{6}$ & Derivative of cinnamic acid & 0.09 \\
\hline 7 & 6.7 & 269,348 & 771.1993 & 609.1451 & 462,301 & $\mathrm{C}_{33} \mathrm{H}_{40} \mathrm{O}_{21}$ & Quercetin 3-rutinoside 7-galactoside & 0.26 \\
\hline 8 & 8.6 & 269,345 & 355.1042 & 161.0227 & 193.179 & $\mathrm{C}_{16} \mathrm{H}_{20} \mathrm{O}_{9}$ & Trans-feruloylglucose & 0.25 \\
\hline 9 & 9.4 & 269,340 & 625.1400 & 316.0221 & 117.163 & $\mathrm{C}_{27} \mathrm{H}_{30} \mathrm{O}_{17}$ & Myricetin-3-rutinoside & 1.91 \\
\hline 10 & 9.5 & 280 & 325.0937 & 119.0497 & 117.163 & $\mathrm{C}_{15} \mathrm{H}_{18} \mathrm{O}_{8}$ & 8 - $\beta$-Glucopyranosyloxycinnamic acid & 3.27 \\
\hline 11 & 9.8 & 266,346 & 623.1262 & 285.0408 & 447 & $\mathrm{C}_{27} \mathrm{H}_{28} \mathrm{O}_{17}$ & Luteolin $4^{\prime}$-glucoside 7-galacturonide & 1.80 \\
\hline 12 & 9.9 & 262,345 & 739.2094 & 284.0327 & $572,255,178$ & $\mathrm{C}_{33} \mathrm{H}_{40} \mathrm{O}_{19}$ & $\begin{array}{c}\text { Kaempferol } \\
\text { 3-(2"-rhamnosylrutinoside) }\end{array}$ & 0.32 \\
\hline 13 & 10.2 & 265,349 & 339.1092 & 145.0276 & 163 & $\mathrm{C}_{16} \mathrm{H}_{20} \mathrm{O}_{8}$ & TPCA $^{1}$ & 0.27 \\
\hline 14 & 10.5 & 256,354 & 609.1462 & 300.0281 & & $\mathrm{C}_{27} \mathrm{H}_{30} \mathrm{O}_{16}$ & Rutin & 18.92 \\
\hline 15 & 10.8 & 269,354 & 463.0912 & 300.0269 & 271,151 & $\mathrm{C}_{21} \mathrm{H}_{20} \mathrm{O}_{12}$ & Quercetin-4'-glucoside & 1.06 \\
\hline 16 & 10.9 & 269,343 & 637.1414 & 299.0567 & 284.2337 & $\mathrm{C}_{28} \mathrm{H}_{30} \mathrm{O}_{17}$ & MHGB $^{2}$ & 2.03 \\
\hline 17 & 11.0 & 264,346 & 447.0930 & 285.0397 & $269,209,251$ & $\mathrm{C}_{21} \mathrm{H}_{20} \mathrm{O}_{11}$ & Kaempferol 3-O-glucoside & 0.64 \\
\hline 18 & 11.4 & 265,344 & 593.1527 & 285.0408 & $151,327,178$ & $\mathrm{C}_{27} \mathrm{H}_{30} \mathrm{O}_{15}$ & Kaempferol 3-O-glucoside ${ }^{3}$ & 1.87 \\
\hline 19 & 11.6 & 254,354 & 623.1626 & 315.0515 & $151,243,271$ & $\mathrm{C}_{28} \mathrm{H}_{32} \mathrm{O}_{16}$ & Isorhamnetin 3-O-rhamnoglucoside & 3.75 \\
\hline
\end{tabular}

${ }^{1}$ Trihydroxy-6-[4-(3-oxobutyl)phenoxy] loxane-2-carboxylic acid; ${ }^{2}$ 2-(3-Methoxy-4-hydroxyphenyl)-5-hydroxy7-[2-O-(beta-D-glucopyranuronosyl)-beta-D-glucopyranosyloxy]-4H-1-benzopyran-4-one; ${ }^{3}$ Kaempferol 3-Oglucoside-7-rhamnoside.

\subsection{Ruminal Fermentation In Vitro}

Methane concentration was significantly lower $(p=0.046)$, but IVDMD $(p<0.001)$, total VFA concentration $(p=0.011)$, and $n$-valerate concentration $(p=0.017)$ were significantly higher, in the SFP than the control group (Table 3). Archaea and Methanomicrobiales populations were smaller in the SFP than the control group $(p<0.001$ and $p=0.009$, respectively).

Table 3. Effect of SFPs on ruminal fermentation and microbial populations in vitro.

\begin{tabular}{ccccc}
\hline & Control & SFP & SEM & $p$ \\
\hline Ammonia N (mg/L) & 270 & 282 & 15.5 & 0.849 \\
Methane (mmoL) & 1.15 & 1.02 & 0.063 & 0.046 \\
pH & 7.60 & 7.45 & 0.041 & 0.064 \\
IVDMD (g/kg DM) & 311 & 447 & 18.0 & $<0.001$ \\
Total VFAs (mmoL/L) & 31.5 & 39.9 & 1.76 & 0.011 \\
Acetate (mol\%) & 65.5 & 66.1 & 0.997 & 0.768 \\
Propionate (mol\%) & 17.7 & 17.1 & 0.799 & 0.711 \\
\hline
\end{tabular}


Table 3. Cont.

\begin{tabular}{ccccc}
\hline & Control & SFP & SEM & $p$ \\
\hline$n$-Butyrate $(\mathrm{mol} \%)$ & 11.0 & 11.2 & 0.239 & 0.764 \\
iso-Butyrate $(\mathrm{mol} \%)$ & 0.861 & 0.617 & 0.158 & 0.455 \\
$n$-Valerate $(\mathrm{mol} \%)$ & 2.25 & 2.56 & 0.066 & 0.017 \\
iso-Valerate $(\mathrm{mol} \%)$ & 2.50 & 2.27 & 0.115 & 0.318 \\
Caproate $(\mathrm{mol} \%)$ & 0.150 & 0.181 & 0.019 & 0.430 \\
Acetate: propionate & 4.02 & 4.10 & 0.353 & 0.923 \\
Total protozoa $\left(10^{3} / \mathrm{mL}\right)$ & 6.65 & 8.7 & 6.612 & 0.438 \\
Total bacteria $\left(10^{8} / \mathrm{mL}\right)$ & 4.02 & 3.9 & 0.164 & 0.721 \\
Archaea $\left(10^{6} / \mathrm{mL}\right)$ & 3.93 & 2.64 & 0.240 & $<0.001$ \\
Methanobacteriales $\left(10^{6} / \mathrm{mL}\right)$ & 1.53 & 1.48 & 0.024 & 0.363 \\
Methanomicrobiales $\left(10^{5} / \mathrm{mL}\right)$ & 1.56 & 1.42 & 0.027 & 0.009 \\
\hline
\end{tabular}

IVDMD, in vitro dry matter digestibility; VFAs, volatile fatty acids.

\subsection{Ruminal Fermentation and Microbiota in the Lambs}

The SFPs significantly affected $(p=0.030)$ methane concentrations in the lambs (Table 4$)$. Total protozoa, expressed as a count per gram of wet RC (wRC) in the lambs, did not differ significantly $(p=0.05)$ between the groups. The populations of Archaea $(p<0.001)$, Methanomicrobiales $(p=0.009)$, and Methanobacteriales $(p<0.001)$ were significantly smaller for the SFP than the control group. The relative abundances of $S$. bovis, B. proteoclasticus, F. succinogenes, M. elsdenii, R. albus, R. flavefaciens, and the genera Prevotella and Lactobacillus did not differ significantly between the groups $(p=0.05)$. The relative abundance of $B$. fibrisolvens was significantly higher in the SFP than in the control group $(p=0.006)$. The relative abundance of the 16S rRNA gene was expressed as an arbitrary unit (AU) relative to the total abundance of bacterial genes of the control group.

Table 4. Effect of SFPs on ruminal fermentation and microbial populations in the lambs.

\begin{tabular}{|c|c|c|c|c|}
\hline & Control & SFP & SD & $p$ \\
\hline Ammonia N (mg/L) & 205 & 199 & 79.1 & 0.849 \\
\hline Methane (mmoL) & 3.17 & 2.30 & 0.903 & 0.030 \\
\hline $\mathrm{pH}$ & 6.36 & 6.37 & 0.575 & 0.974 \\
\hline Total VFAs (mmoL/L) & 68.2 & 70.2 & 27.71 & 0.847 \\
\hline Acetate $(\mathrm{mol} \%)$ & 74.6 & 73.3 & 4.33 & 0.462 \\
\hline Propionate (mol\%) & 11.9 & 10.5 & 4.42 & 0.425 \\
\hline n-Butyrate (mol\%) & 11.1 & 13.5 & 3.96 & 0.123 \\
\hline iso-Butyrate (mol\%) & 0.06 & 0.06 & 0.140 & 0.946 \\
\hline n-Valerate (mol\%) & 1.33 & 1.60 & 0.828 & 0.414 \\
\hline iso-Valerate $(\mathrm{mol} \%)$ & 0.89 & 0.77 & 0.595 & 0.633 \\
\hline Caproate (mol\%) & 0.20 & 0.34 & 0.473 & 0.457 \\
\hline Acetate: Propionate & 7.19 & 7.91 & 0.529 & 0.507 \\
\hline Total protozoa $\left(10^{5} / \mathrm{g}\right.$ wRC) & 6.7 & 8.2 & 5.61 & 0.508 \\
\hline Archaea $\left(10^{7} / \mathrm{mL}\right)$ & 7.71 & 4.79 & 1.84 & $<0.001$ \\
\hline Methanobacteriales $\left(10^{7} / \mathrm{mL}\right)$ & 3.74 & 2.90 & 0.672 & $<0.001$ \\
\hline Methanomicrobiales $\left(10^{7} / \mathrm{mL}\right)$ & 3.50 & 2.50 & 0.463 & 0.009 \\
\hline Total bacteria (AU) & 1.0 & 1.01 & 0.110 & 0.863 \\
\hline S. bovis $(\mathrm{AU})$ & 1.0 & 1.04 & 0.145 & 0.525 \\
\hline B. proteoclasticus (AU) & 1.0 & 1.06 & 0.161 & 0.325 \\
\hline B. fibrisolvens (AU) & 1.0 & 1.13 & 0.124 & 0.006 \\
\hline F. succinogenes (AU) & 1.0 & 0.97 & 0.311 & 0.832 \\
\hline M. elsdenii (AU) & 1.0 & 0.94 & 0.258 & 0.587 \\
\hline R. albus (AU) & 1.0 & 1.10 & 0.175 & 0.160 \\
\hline R. flavefaciens (AU) & 1.0 & 0.97 & 0.147 & 0.594 \\
\hline Genus Prevotella (AU) & 1.0 & 0.96 & 0.299 & 0.729 \\
\hline Genus Lactobacillus (AU) & 1.0 & 1.04 & 0.126 & 0.500 \\
\hline
\end{tabular}

wRC, count per gram of wet ruminal content; AU, arbitrary unit. 


\subsection{Hematological Parameters}

The complete red blood cell (RBC) hemograms of each infected animal identified clinical signs of haemonchosis such as anemia after D23 (Table 5). The RBC count, level of hemoglobin (HGB), hematocrit (HCT), and mean corpuscular volume (MCV) were not significantly influenced by treatment or the interaction between treatment and time $(p=0.05)$. Time significantly affected all RBC parameters $(p<0.001)$. Total leukocytes, neutrophils, and lymphocytes were not significantly influenced by treatment, time, or the interaction between them $(p=0.05$, Table 6$)$. Treatment and time significantly affected the counts of monocytes $(p=0.05)$ and basophils ( $p=0.05$ and $p=0.01$, respectively), and time significantly affected the counts of eosinophils $(p<0.001)$.

Table 5. Effects of SFPs on the red blood cell parameters of the lambs.

\begin{tabular}{|c|c|c|c|c|c|c|c|}
\hline & \multirow{2}{*}{ Day } & \multirow{2}{*}{ Control } & \multirow{2}{*}{ SFP } & \multirow{2}{*}{ SD } & \multicolumn{3}{|c|}{$p$} \\
\hline & & & & & Treatment (T) & Time & $\mathbf{T} \times$ Time \\
\hline \multirow{5}{*}{$\begin{array}{l}\mathrm{RBC} \\
(\mathrm{T} / \mathrm{L})\end{array}$} & 0 & 11.37 & 11.63 & 1.176 & \multirow{5}{*}{0.133} & \multirow{5}{*}{$<0.001$} & \multirow{5}{*}{0.681} \\
\hline & 23 & 7.68 & 7.10 & 1.289 & & & \\
\hline & 30 & 7.22 & 6.56 & 1.382 & & & \\
\hline & 37 & 7.15 & 6.55 & 1.373 & & & \\
\hline & 44 & 7.00 & 6.83 & 1.276 & & & \\
\hline \multirow{5}{*}{$\begin{array}{l}\text { Hemoglobin } \\
\qquad(\mathrm{g} / \mathrm{L})\end{array}$} & 0 & 111.7 & 115.5 & 11.62 & \multirow{5}{*}{0.326} & \multirow{5}{*}{$<0.001$} & \multirow{5}{*}{0.462} \\
\hline & 23 & 74.6 & 68.9 & 11.30 & & & \\
\hline & 30 & 71.5 & 64.8 & 12.89 & & & \\
\hline & 37 & 72.0 & 67.1 & 14.28 & & & \\
\hline & 44 & 69.5 & 71.7 & 14.29 & & & \\
\hline \multirow{5}{*}{$\begin{array}{l}\text { Hematocrit } \\
\qquad(\mathrm{g} / \mathrm{L})\end{array}$} & 0 & 0.263 & 0.265 & 0.0251 & \multirow{5}{*}{0.208} & \multirow{5}{*}{$<0.001$} & \multirow{5}{*}{0.458} \\
\hline & 23 & 0.187 & 0.171 & 0.0261 & & & \\
\hline & 30 & 0.182 & 0.165 & 0.0313 & & & \\
\hline & 37 & 0.185 & 0.174 & 0.0339 & & & \\
\hline & 44 & 0.178 & 0.187 & 0.0336 & & & \\
\hline \multirow{5}{*}{$\begin{array}{c}\mathrm{MCV} \\
(\mathrm{fL})\end{array}$} & 0 & 23.2 & 23.0 & 1.86 & \multirow{5}{*}{0.281} & \multirow{5}{*}{$<0.001$} & \multirow{5}{*}{0.354} \\
\hline & 23 & 24.5 & 24.4 & 2.11 & & & \\
\hline & 30 & 25.4 & 25.3 & 2.25 & & & \\
\hline & 37 & 26.0 & 26.7 & 2.04 & & & \\
\hline & 44 & 25.6 & 27.4 & 2.29 & & & \\
\hline
\end{tabular}

RBC, red blood cell; $\mathrm{T} / \mathrm{L}, 10^{12}$ per liter; $\mathrm{MCV}$, mean corpuscular volume; SD, standard deviation.

Table 6. Effects of SFPs on the total leukocyte and differential counts of the lambs.

\begin{tabular}{|c|c|c|c|c|c|c|c|}
\hline & \multirow{2}{*}{ Day } & \multirow{2}{*}{ Control } & \multirow{2}{*}{ SFP } & \multirow{2}{*}{ SD } & \multicolumn{3}{|c|}{$p$} \\
\hline & & & & & Treatment (T) & Time & $\mathrm{T} \times$ Time \\
\hline \multirow{5}{*}{$\begin{array}{c}\text { Total } \\
\text { leukocyte } \\
(\mathrm{G} / \mathrm{L})\end{array}$} & 0 & 8.68 & 8.92 & 2.280 & \multirow{5}{*}{0.159} & \multirow{5}{*}{0.225} & \multirow{5}{*}{0.951} \\
\hline & 23 & 8.00 & 9.25 & 2.515 & & & \\
\hline & 30 & 7.24 & 7.81 & 4.475 & & & \\
\hline & 37 & 7.29 & 7.71 & 1.705 & & & \\
\hline & 44 & 6.55 & 7.90 & 2.047 & & & \\
\hline \multirow{5}{*}{$\begin{array}{c}\text { Neutrophils } \\
\text { (G/L) }\end{array}$} & 0 & 3.18 & 3.10 & 1.662 & \multirow{5}{*}{0.361} & \multirow{5}{*}{0.304} & \multirow{5}{*}{0.923} \\
\hline & 23 & 3.13 & 3.66 & 1.589 & & & \\
\hline & 30 & 2.69 & 2.84 & 1.064 & & & \\
\hline & 37 & 2.63 & 2.68 & 1.095 & & & \\
\hline & 44 & 2.51 & 3.01 & 1.526 & & & \\
\hline
\end{tabular}


Table 6. Cont.

\begin{tabular}{|c|c|c|c|c|c|c|c|}
\hline & \multirow{2}{*}{ Day } & \multirow{2}{*}{ Control } & \multirow{2}{*}{ SFP } & \multirow{2}{*}{ SD } & \multicolumn{3}{|c|}{$p$} \\
\hline & & & & & Treatment (T) & Time & $\mathrm{T} \times$ Time \\
\hline \multirow{5}{*}{$\begin{array}{c}\text { Lymphocytes } \\
(\mathrm{G} / \mathrm{L})\end{array}$} & 0 & 2.23 & 2.22 & 1.313 & \multirow{5}{*}{0.899} & \multirow{5}{*}{0.606} & \multirow{5}{*}{0.987} \\
\hline & 23 & 1.95 & 2.13 & 0.977 & & & \\
\hline & 30 & 2.35 & 2.19 & 1.045 & & & \\
\hline & 37 & 2.61 & 2.50 & 1.186 & & & \\
\hline & 44 & 2.21 & 2.18 & 1.214 & & & \\
\hline \multirow{5}{*}{$\begin{array}{c}\text { Monocytes } \\
(\mathrm{G} / \mathrm{L})\end{array}$} & 0 & 2.38 & 2.55 & 1.288 & \multirow{5}{*}{0.047} & \multirow{5}{*}{0.024} & \multirow{5}{*}{0.977} \\
\hline & 23 & 2.10 & 2.48 & 1.055 & & & \\
\hline & 30 & 1.87 & 2.30 & 0.983 & & & \\
\hline & 37 & 1.67 & 1.92 & 0.606 & & & \\
\hline & 44 & 1.44 & 1.93 & 0.686 & & & \\
\hline \multirow{5}{*}{$\begin{array}{l}\text { Eosinophils } \\
(\mathrm{G} / \mathrm{L})\end{array}$} & 0 & 0.093 & 0.096 & 0.0596 & \multirow{5}{*}{0.200} & \multirow{5}{*}{$<0.001$} & \multirow{5}{*}{0.395} \\
\hline & 23 & 0.046 & 0.041 & 0.0193 & & & \\
\hline & 30 & 0.065 & 0.033 & 0.0356 & & & \\
\hline & 37 & 0.043 & 0.033 & 0.0177 & & & \\
\hline & 44 & 0.039 & 0.041 & 0.0225 & & & \\
\hline \multirow{5}{*}{$\begin{array}{l}\text { Basophils } \\
\text { (G/L) }\end{array}$} & 0 & 0.803 & 0.945 & 0.7947 & \multirow{5}{*}{0.028} & \multirow{5}{*}{0.002} & \multirow{5}{*}{0.930} \\
\hline & 23 & 0.778 & 0.953 & 0.6667 & & & \\
\hline & 30 & 0.275 & 0.445 & 0.2770 & & & \\
\hline & 37 & 0.336 & 0.575 & 0.4151 & & & \\
\hline & 44 & 0.349 & 0.751 & 0.5041 & & & \\
\hline
\end{tabular}

$\mathrm{SD}$, standard deviation; $\mathrm{G} / \mathrm{L}, 10^{9}$ per liter.

\subsection{Histopathology}

The changes in the abomasal tissues of the lambs inoculated with $H$. contortus were mild and included damage to epithelial cells and inflammatory infiltration (Table 7). The percentage of damaged glands $(p=0.004)$ was significantly higher, and mucosal hypertrophy (not significant, $p=0.05$ ) was marginally higher, in the SFP than in the control group. Glandular dilatation and submucosal edema were similar in both groups.

Table 7. Effects of SFPs on the histopathology of the abomasal tissues of the lambs.

\begin{tabular}{ccccc}
\hline Effect & Control & SFP & SD & $p$ \\
\hline Hypertrophy of mucosa (\%) & 15.4 & 30.8 & 3.31 & 0.375 \\
Epithelial cell damage (\%) & 100 & 100 & 0.00 & - \\
Hyperplasia of mucus-producing cells (\%) & 7.7 & 0 & 1.51 & 0.327 \\
Dilatation of glands (\%) & 30.8 & 30.8 & 3.62 & 0.999 \\
Damage of glands (\%) & 0 & 46.2 & 3.31 & 0.004 \\
Inflammatory cell infiltration (\%) & 100 & 100 & 0.00 & - \\
Submucosal edema (\%) & 30.8 & 38.5 & 3.73 & 0.695 \\
\hline
\end{tabular}

\section{Discussion}

Multidisciplinary studies (agronomic, nutritional, parasitological, and chemical) have identified many benefits to animal health of tanniferous forages and legumes used as feed for ruminants [40,41]. Sainfoin generally contains mainly CTs (approximately $42-50 \mathrm{~g}$ CTs/kg DM), which have been well studied [42-44]. The consumption of sainfoin disturbs various stages of parasitic life cycles, mainly due to its high tannin content [45]. The anthelmintic activity of CTs, however, can be increased by the addition of flavonoids, which also interfere with the biology of GINs $[15,16]$. We therefore focused on the analysis of flavonoids and phenolic acids.

Quantitative UHRMS analyses of the bioactive components in the SFPs identified more than $32.0 \mathrm{~g} / \mathrm{kg}$ DM flavonoids and $4.5 \mathrm{~g} / \mathrm{kg}$ DM phenolic acids. The main flavonoid was rutin $(18.92 \mathrm{mg} / \mathrm{g} \mathrm{DM})$, which has multiple pharmacological activities with metabolic 
health benefits [46]. Rutin can alter the ruminal microbiome and reduce the population of methanogenic bacteria [47]; and adding rutin $(3.0 \mathrm{mg} / \mathrm{kg})$ to feed dairy cows for 11 weeks improved the efficiency of carbohydrate fermentation in the rumen and the ability to synthesize protein [48]. Our results with SFPs indicated reductions in methane concentrations and Archaea population sizes of 11 and $33 \%$ and 27 and $38 \%$ in in vitro and in vivo treatments, respectively. Ruminal contents in the SFP group indicated also smaller populations of Methanomicrobiales (in vitro and in vivo) and Methanobacteriales (in vivo) than the control group. In another study, dietary supplementation with dry fumitory, mallow, wormwood, and chamomile with flavonoids $(0.4-12.2 \mathrm{~g} / \mathrm{kg} \mathrm{DM})$ for $70 \mathrm{~d}$ had no antimethanogenic effect in lambs [19]. The anti-methanogenic effect of SFPs observed in our study may have been due to the direct effect of either the CTs [6,49] or rutin [50] or both on methanogenesis in the SFP group. Data on reducing methane concentration by rutin supplementation in vitro and in vivo, however, have been inconsistent [51-53]. Acceptably low methane emissions can therefore be achieved by a suitable choice of the vegetative stage of sainfoin [54]. The stimulation of gas concentration by rutin $(50 \mathrm{mg} / \mathrm{g} \mathrm{DM})$ in ruminal fermentation in vitro led to an increased $\mathrm{CO}_{2}$ concentration and a decreased methane concentration, probably because rutin is a substrate for nonmethanogenic microbiota [50]. However, the consumption of SFPs by infected lambs for $14 \mathrm{~d}$ in our experiment affected ruminal methanogens and consequently reduced methane emission without adverse changes in the ruminal microbiome.

The relative abundance of $B$. fibrisolvens in our study was higher in the SFP than the control group, because the replacement of MH by SFPs was probably associated with the increased demand for microbial degradation of fiber in the SFP group. Other bacterial species were not significantly affected, probably due to the relatively short SFP treatment. B. fibrisolvens plays an important role in the ruminal fermentation of polysaccharides that participate in cellulolytic processes in the rumen but do not have an autonomous cellulolytic capacity [55]. Changes in the relative abundance of $B$. fibrisolvens suggest that the SFPs did not negatively affect ruminal fermentation (e.g., total and individual VFAs). A diet with plant bioactive compounds can affect the ruminal microbiome, the kinetics of fermentation, and the response and adaptation to anti-methanogenic compounds, sometimes leading to inconsistent efficacy of phytochemicals [56-58]. We cannot sufficiently confirm the effect of SFPs on ruminal fermentation in lambs, because the SFPs were consumed for only $14 \mathrm{~d}$. We also observed no effects of short-term SFP feeding on total protozoal counts in vitro and in vivo. The in vitro experiments on the effects of $\mathrm{CT}$ fractions of differing molecular weights on Leucaena leucocephala identified a lowering of the total number of ciliate protozoa with changes in counts of community members [59]. Similar effects were observed in vivo after long-term feeding of lambs with extracts of Acacia negra and Uncaria gambir, sources rich in CTs [60]. The feeding of dry leaves of L. leucocephala (12-36\% of DM intake), however, did not affect ruminal protozoan, bacterial, or archaeal populations in crossbred heifers [61]. Supplementation of rutin for three weeks in dairy cows $(3.0 \mathrm{mg} / \mathrm{kg}$ diet) did not significantly decrease counts of ruminal protozoa [49]. The amount and composition of the CTs and the length of treatment are likely the main factors influencing the effects of CT on the ruminal microbiome. In our experiment, even feeding only SFPs did not affect the protozoan counts. We can speculate about the amount of intake of CTs in the diet of the control group. Several studies have reported the CT compositions of various kinds of forages and $\mathrm{MH}$ from permanent pastures [6,62]. Unfortunately, we did not measure the $\mathrm{CT}$ content of the control $\mathrm{MH}$ diet.

Our study confirmed a significant reduction in RBC count, HGB level, and HCT from D23 in both groups of lambs infected with $H$. contortus, consistent with our previous results $[20,28,63]$. The reductions were likely due to damage caused by the GIN parasites, but SFPs as a replacement for hay did not affect the RBC parameters. The intensity and duration of hematological disorders depend on the nutritional status of infected sheep because protein-enriched diets induce resistance to infection associated with the improved regenerative capacity of bone marrow [64]. In addition to the nutritional status of the 
host, differences in RBC disorders during GIN infection may be affected by the species of nematode, the severity of the infection, the iron stores and bodily reserves of the host, and the susceptibility of the host breed [65]. The basophil level in our study was higher in the infected lambs of the SFP group. Basophils are generally relatively rare and short-lived cells and probably played an important role in the immune response in the SFP group to GIN infection in our experiment [66]. The number of monocytes, eosinophils, and basophils was differentially affected by the time after infection, consistent with the blood variables during a subclinical $H$. contortus infection [67].

The development of GINs in host abomasa causes pathology, with mucosal damage and gastropathy with protein loss, followed by inflammatory immune responses of the host [68]. Our observations included microscopic changes in the abomasa, such as mucosal hypertrophy, damage to epithelial cells, mucus-producing cell hyperplasia, glandular dilatation, glandular damage, inflammatory cell infiltration, and submucosal edema. A roughened and hyperemic abomasal mucosa with enlarged glands and globular leukocytes have been described in lambs infected with $H$. contortus [69]. In our experiment, glandular damage in the SFP group differed significantly between the experimental groups, but the other changes in the abomasal mucosa were essentially the same for both infected groups. Mucosal hypertrophy was also more pronounced but not significant in the lambs fed with SFPs. An increased percentage of abomasal injuries and mucosal hypertrophy in the SFP group was attributed to regeneration, which is more common in abomasal tissue due to herbal treatment [70]. The histopathological changes in lambs in our previous study infected with $H$. contortus and supplemented with dry Artemisia absinthium and Malva sylvestris were predominantly on the mucosal membrane, with inflammatory cell infiltrates (mainly lymphocytes and macrophages with a mixture of eosinophils, plasma cells, and mast cells) [70]. Subclinical $H$. contortus infections generally damage the abomasal mucosa, which was very similar in the two infected groups, i.e., with and without SFPs.

\section{Conclusions}

Replacing MH with SFPs affected the composition of methanogenic bacteria in the rumens of the lambs and consequently reduced methane emissions, thus helping to reduce the environmental burden of methane and minimizing the adverse effects on animal health. From this point of view, the use of SFPs can be very useful in good agricultural practice. This study, to the best of our knowledge, is the first on the effect of SFPs on the ruminal microbiome and the abomasal histopathology of lambs loaded with parasites.

Author Contributions: Conceptualization, Z.V. and D.P.; methodology, D.P.; software, S.Ś.; validation, D.P., D.B. and M.K. (Martyna Kozłowska); formal analysis, A.E. and W.F.; investigation, D.P.; resources, D.P. and M.K. (Michaela Komáromyová); data curation, A.C., M.S.-S. and S.K.; writing—original draft preparation, D.P.; writing—review and editing, Z.V.; supervision, Z.V.; project administration, Z.V. and M.V.; funding acquisition, M.V. All authors have read and agreed to the published version of the manuscript.

Funding: This study was supported by funds from the Slovak Research and Development Agency (www.apvv.sk accessed on 1 July 2019) (APVV 18-0131), the SAS Programme for PhD student grants (www.doktogrant.sav.sk accessed on 1 June 2020) (DoktoGrant APP0162) and the Polish Ministry of Science and Higher Education "Regional Initiative Excellence" program 2019-2022, Project No. 005/RID/2018/19.

Institutional Review Board Statement: The study was conducted according to the guidelines of the Declaration of Helsinki and approved by the Ethical Committee of the Institute of Parasitology of the Slovak Academy of Sciences on 22 November 2020 (protocol code 2020/21).

Informed Consent Statement: Not applicable.

Data Availability Statement: Data are available upon reasonable request to the corresponding author. 


\begin{abstract}
Acknowledgments: The English has been revised throughout the manuscript by a native English language editor, William Blackhall. The authors are grateful to Valéria Venglovská, Peter Jerga, and Gabriel Benkovský for their technical support.

Conflicts of Interest: The authors declare no conflict of interest. The funders had no role in the design of the study; in the collection, analyses, or interpretation of data; in the writing of the manuscript, or in the decision to publish the results.
\end{abstract}

\title{
References
}

1. Houdijk, J.G.M.; Tolkamp, B.J.; Rooke, J.A.; Hutchings, M.R. Animal health and greenhouse gas intensity: The paradox of periparturient parasitism. Int. J. Parasitol. 2017, 47, 633-641. [CrossRef] [PubMed]

2. Fox, N.J.; Smith, L.A.; Houdijk, J.G.M.; Athanasiadou, S.; Hutchings, M.R. Ubiquitous parasites drive a $33 \%$ increase in methane yield from livestock. Int. J. Parasitol. 2018, 48, 1017-1021. [CrossRef] [PubMed]

3. Mravčáková, D.; Váradyová, Z.; Kopčáková, A.; Čobanová, K.; Grešáková, L.; Kišidayová, S.; Babják, M.; Urda Dolinská, M.; Dvorožňáková, E.; Königová, A.; et al. Natural chemotherapeutic alternatives for controlling of haemonchosis in sheep. BMC Vet. Res. 2019, 15, 302. [CrossRef] [PubMed]

4. Verma, S.; Taube, F.; Malisch, C.S. Examining the variables leading to apparent incongruity between antimethanogenic potential of tannins and their observed effects in ruminants-A review. Sustainability 2021, 13, 2743. [CrossRef]

5. Hassan, F.; Arshad, M.A.; Ebeid, H.M.; Rehman, M.S.; Khan, M.S.; Shahid, S.; Yang, C. Phytogenic additives can modulate rumen microbiome to mediate fermentation kinetics and methanogenesis through exploiting diet-microbe interaction. Front. Vet. Sci. 2020, 7, 575801. [CrossRef]

6. Waghorn, G. Beneficial and detrimental effects of dietary condensed tannins for sustainable sheep and goat production-Progress and challenges. Anim. Feed Sci. Technol. 2008, 147, 116-139. [CrossRef]

7. Quijada, J.; Fryganas, C.; Ropiak, H.M.; Ramsay, A.; Mueller-Harvey, I.; Hoste, H. Anthelmintic activities against Haemonchus contortus or Trichostrongylus colubriformis from small ruminants are influenced by structural features of condensed tannins. J. Agric. Food Chem. 2015, 63, 6346-6354. [CrossRef]

8. Kommuru, D.S.; Barker, T.; Desai, S.; Burke, J.M.; Ramsay, A.; Mueller-Harvey, I.; Miller, J.E.; Mosjidis, J.A.; Kamisetti, N.; Terrill, T.H. Use of pelleted sericea lespedeza (Lespedeza cuneata) for natural control of coccidia and gastrointestinal nematodes in weaned goats. Vet. Parasitol. 2014, 204, 191-198. [CrossRef]

9. Mechineni, A.; Kommuru, D.S.; Gujja, S.; Mosjidis, J.A.; Miller, J.E.; Burke, J.M.; Ramsay, A.; Mueller-Harvey, I.; Kannan, G.; Lee, J.H.; et al. Effect of fall-grazed sericea lespedeza (Lespedeza cuneata) on gastrointestinal nematode infections of growing goats. Vet. Parasitol. 2014, 204, 221-228. [CrossRef]

10. Hatew, B.; Stringano, E.; Mueller-Harvey, I.; Hendriks, W.H.; Carbonero, C.H.; Smith, L.M.J.; Pellikaan, W.F. Impact of variation in structure of condensed tannins from sainfoin (Onobrychis viciifolia) on in vitro ruminal methane production and fermentation characteristics. J. Anim. Physiol. Anim. Nutr. 2016, 100, 348-360. [CrossRef]

11. Waghorn, G.C.; Ulyatt, M.J.; John, A.; Fisher, M.T. The effect of condensed tannins on the site of digestion of amino acids and other nutrients in sheep fed on Lotus corniculatus L. Br. J. Nutr. 1987, 57, 115-126. [CrossRef] [PubMed]

12. Wang, Y.; Waghorn, G.C.; McNabb, W.C.; Barry, T.N.; Hedley, M.J.; Shelton, I.D. Effect of condensed tannins in Lotus corniculatus upon the digestion of methionine and cysteine in the small intestine of sheep. J. Agric. Sci. 1996, 127, 413-421. [CrossRef]

13. Brunet, S.; Fourquaux, I.; Hoste, H. Ultrastructural changes in the third-stage, infective larvae of ruminant nematodes treated with sainfoin (Onobrychis viciifolia) extract. Parasitol. Int. 2011, 60, 419-424. [CrossRef]

14. Desrues, O.; Peña-Espinoza, M.; Hansen, T.V.; Enemark, H.L.; Thamsborg, S.M. Anti-parasitic activity of pelleted sainfoin (Onobrychis viciifolia) against Ostertagia ostertagi and Cooperia oncophora in calves. Parasit. Vectors 2016, 9, 329. [CrossRef] [PubMed]

15. Barrau, E.; Fabre, N.; Fouraste, I.; Hoste, H. Effect of bioactive compounds from sainfoin (Onobrychis viciifolia Scop.) on the in vitro larval migration of Haemonchus contortus: Role of tannins and flavonol glycosides. Parasitology 2005, 131, 531-538. [CrossRef] [PubMed]

16. Klongsiriwet, C.; Quijada, J.; Williams, A.R.; Mueller-Harvey, I.; Williamson, E.M.; Hoste, H. Synergistic inhibition of Haemonchus contortus exsheathment by flavonoid monomers and condensed tannins. Int. J. Parasitol. Drugs Drug Resist. 2015, 5, 127-134. [CrossRef]

17. Gaudin, E.; Simon, M.; Quijada, J.; Schelcher, F.; Sutra, J.-F.; Lespine, A.; Hoste, H. Efficacy of sainfoin (Onobrychis viciifolia) pellets against multi resistant Haemonchus contortus and interaction with oral ivermectin: Implications for on-farm control. Vet. Parasitol. 2016, 227, 122-129. [CrossRef]

18. Petrič, D.; Mravčáková, D.; Kucková, K.; Čobanová, K.; Kišidayová, S.; Cieslak, A.; Slusarczyk, S.; Váradyová, Z. Effect of dry medicinal plants (wormwood, chamomile, fumitory and mallow) on in vitro ruminal antioxidant capacity and fermentation patterns of sheep. J. Anim. Physiol. Anim. Nutr. 2020, 104, 1219-1232. [CrossRef]

19. Petrič, D.; Mravčáková, D.; Kucková, K.; Kišidayová, S.; Cieslak, A.; Szumacher-Strabel, M.; Huang, H.; Kolodziejski, P.; Lukomska, A.; Slusarczyk, S.; et al. Impact of zinc and/or herbal mixture on ruminal fermentation, microbiota, and histopathology in lambs. Front. Vet. Sci. 2021, 8, 630971. [CrossRef] 
20. Váradyová, Z.; Mravčáková, D.; Babják, M.; Bryszak, M.; Grešáková, L'.; Čobanová, K.; Kišidayová, S.; Plachá, I.; Königová, A.; Cieslak, A.; et al. Effects of herbal nutraceuticals and/or zinc against Haemonchus contortus in lambs experimentally infected. BMC Vet. Res. 2018, 14, 78. [CrossRef]

21. Coles, G.C.; Bauer, C.; Borgsteede, F.H.M.; Geerts, S.; Klei, T.R.; Taylor, M.A.; Waller, P.J. World Association for the Advancement of Veterinary Parasitology (W.A.A.V.P) methods for the detection of anthelmintic resistance in nematodes of veterinary importance. Vet. Parasitol. 1992, 44, 35-44. [CrossRef]

22. European Commission (EC). Council Regulation (EC) 1099/2009 of 24 September 2009 on the Protection of Animals at the Time of Killing. Available online: https:/ / eur-lex.europa.eu/legal-content/EN/TXT/?uri=celex:32009R1099 (accessed on 18 November 2009).

23. Yánez-Ruiz, D.R.; Bannink, A.; Dijkstra, J.; Kebreab, E.; Morgavi, D.P.; O’Kiely, P.; Reynolds, C.K.; Schwarm, A.; Shingfield, K.J.; $\mathrm{Yu}, \mathrm{Z}$.; et al. Design, implementation and interpretation of in vitro batch culture experiments to assess enteric methane mitigation in ruminants-A review. Anim. Feed Sci. Technol. 2016, 216, 1-18. [CrossRef]

24. McDougall, E.I. Studies on ruminant saliva. I. The composition and output of sheep's saliva. Biochem. J. 1948, 43, 99-109. [CrossRef] [PubMed]

25. Horwitz, W. Official Methods of AOAC International, 17th ed.; Association of Official Analytical Chemists (AOAC) International: Gaithersburg, MD, USA; Washington, DC, USA, 2000.

26. Van Soest, P.J.; Robertson, J.B.; Lewis, B.A. Methods for dietary fiber neutral detergent fiber, and non-starch polysaccharides in relation to animal nutrition. J. Dairy Sci. 1991, 74, 3583-3597. [CrossRef]

27. Mravčáková, D.; Komáromyová, M.; Babják, M.; Urda Dolinská, M.; Königová, A.; Petrič, D.; Čobanová, K.; Ślusarczyk, S.; Cieslak, A.; Várady, M.; et al. Anthelmintic activity of wormwood (Artemisia absinthium L.) and mallow (Malva sylvestris L.) against Haemonchus contortus in sheep. Animals 2020, 10, 219. [CrossRef]

28. Váradyová, Z.; Kišidayová, S.; Čobanová, K.; Grešákova, L’.; Babják, M.; Königova, A.; Urda Dolinská, M.; Várady, M. The impact of a mixture of medicinal herbs on ruminal fermentation, parasitological status and hematological parameters of the lambs experimentally infected with Haemonchus contortus. Small Rumin. Res. 2017, 151, 124-132. [CrossRef]

29. Wolin, M.J. A Theoretical Rumen Fermentation Balance. J. Dairy Sci. 1960, 43, 1452-1459. [CrossRef]

30. Broderick, G.A.; Kang, J.H. Automated simultaneous determination of ammonia and total amino acids in ruminal fluid and in vitro media. J. Dairy Sci. 1980, 63, 64-75. [CrossRef]

31. Williams, A.G.; Coleman, G.S. The Rumen Protozoa, 1st ed.; Springer: New York, NY, USA, 1992; pp. 1-425.

32. Szulc, P.; Mravčáková, D.; Szumacher-Strabel, M.; Váradyová, Z.; Várady, M.; Čobanová, K.; Syahrulawal, L.; Patra, A.K.; Cieslak, A. Ruminal fermentation, microbial population and lipid metabolism in gastrointestinal nematode-infected lambs fed a diet supplemented with herbal mixtures. PLoS ONE 2020, 15, e0231516. [CrossRef]

33. Henderson, G.; Cox, F.; Kittelmann, S.; Miri, V.H.; Zethof, M.; Noel, S.J.; Waghorn, G.C.; Janssen, P.H. Effect of DNA extraction methods and sampling techniques on the apparent structure of cow and sheep rumen microbial communities. PLoS ONE 2013, 8, e74787. [CrossRef]

34. Stevenson, D.M.; Weimer, P.J. Dominance of Prevotella and low abundance of classical ruminal bacterial species in the bovine rumen revealed by relative quantification real-time PCR. Appl. Microbiol. Biotechnol. 2007, 75, 165-174. [CrossRef] [PubMed]

35. Potu, R.B.; AbuGhazaleh, A.A.; Hastings, D.; Jones, K.; Ibrahim, S.A. The effect of lipid supplements on ruminal bacteria in continuous culture fermenters varies with the fatty acid composition. J. Microbiol. 2011, 49, 216-223. [CrossRef] [PubMed]

36. Li, M.; Penner, G.B.; Hernandez-Sanabria, E.; Oba, M.; Guan, L.L. Effects of sampling location and time, and host animal on assessment of bacterial diversity and fermentation parameters in the bovine rumen. J. Appl. Microbiol. 2009, 107, 1924-1934. [CrossRef] [PubMed]

37. Denman, S.E.; McSweeney, C.S. Development of a real-time PCR assay for monitoring anaerobic fungal and cellulolytic bacterial populations within the rumen. FEMS Microbiol. Ecol. 2006, 58, 572-582. [CrossRef]

38. Wang, R.F.; Beggs, M.L.; Erickson, B.D.; Cerniglia, C.E. DNA microarray analysis of predominant human intestinal bacteria in fecal samples. Mol. Cell Probes. 2004, 18, 223-234. [CrossRef]

39. Maeda, H.; Fujimoto, C.; Haruki, Y.; Maeda, T.; Kokeguchi, S.; Petelin, M.; Arai, H.; Tanimoto, I.; Nishimura, F.; Takashiba, S. Quantitative real-time PCR using TaqMan and SYBR Green for Actinobacillus actinomycetemcomitans, Porphyromonas gingivalis, Prevotella intermedia, tetQ gene and total bacteria. FEMS Immunol. Med. Microbiol. 2003, 39, 81-86. [CrossRef]

40. Mueller-Harvey, I.; Bee, G.; Dohme-Meier, F.; Hoste, H.; Karonen, M.; Kölliker, R.; Lüscher, A.; Niderkorn, V.; Pellikaan, W.F.; Salminen, J.-P.; et al. Benefits of condensed tannins in forage legumes fed to ruminants: Importance of structure, concentration, and diet composition. Crop Sci. 2019, 59, 861-885. [CrossRef]

41. Niderkorn, V.; Jayanegara, A. Opportunities offered by plant bioactive compounds to improve silage quality, animal health and product quality for sustainable ruminant production: A review. Agronomy 2021, 11, 86. [CrossRef]

42. Malisch, C.S.; Lüscher, A.; Baert, N.; Engström, M.T.; Studer, B.; Fryganas, C.; Suter, D.; Mueller-Harvey, I.; Salminen, J.-P. Large variability of proanthocyanidin content and composition in sainfoin (Onobrychis viciifolia). J. Agric. Food Chem. 2015, 63, 10234-10242. [CrossRef]

43. Wang, Y.; McAllister, T.A.; Acharya, S. Condensed tannins in sainfoin: Composition, concentration, and effects on nutritive and feeding value of sainfoin forage. Crop Sci. 2015, 55, 13-22. [CrossRef] 
44. Rivaroli, D.; Prunier, A.; Meteau, K.; do Prado, I.N.; Prache, S. Tannin-rich sainfoin pellet supplementation reduces fat volatile indoles content and delays digestive parasitism in lambs grazing alfalfa. Animal 2019, 13, 1883-1890. [CrossRef] [PubMed]

45. Hoste, H.; Torres-Acosta, J.F.; Sandoval-Castro, C.A.; Mueller-Harvey, I.; Sotiraki, S.; Louvandini, H.; Thamsborg, S.M.; Terrill, T.H. Tannin containing legumes as a model for nutraceuticals against digestive parasites in livestock. Vet. Parasitol. 2015, 212, 5-17. [CrossRef]

46. Ganeshpurkar, A.; Saluja, A.K. The pharmacological potential of rutin. Saudi Pharm. J. 2017, 25, 149-164. [CrossRef] [PubMed]

47. Santra, A.; Saikia, A.; Baruah, K.K. Scope of rumen manipulation using medicinal plants to mitigate methane production. J. Pharmacogn. 2012, 3, 115-120.

48. Cui, K.; Guo, X.D.; Tu, Y.; Zhang, N.F.; Ma, T.; Diao, Q.Y. Effect of dietary supplementation of rutin on lactation performance, ruminal fermentation and metabolism in dairy cows. J. Anim. Physiol. Anim. Nutr. 2015, 99, 1065-1073. [CrossRef] [PubMed]

49. Niderkorn, V.; Barbier, E.; Macheboeuf, D.; Torrent, A.; Mueller-Harvey, I.; Hoste, H. In vitro rumen fermentation of diets with different types of condensed tannins derived from sainfoin (Onobrychis viciifolia Scop.) pellets and hazelnut (Corylus avellana L.) pericarps. Anim. Feed Sci. Technol. 2020, 259, 114357. [CrossRef]

50. Leiber, F.; Kunz, C.; Kreuzer, M. Influence of different morphological parts of buckwheat (Fagopyrum esculentum) and its major secondary metabolite rutin on rumen fermentation in vitro. Czech J. Anim. Sci. 2012, 57, 10-18. [CrossRef]

51. Oskoueian, E.; Abdullah, N.; Oskoueian, A. Effects of flavonoids on rumen fermentation activity, methane production, and microbial population. Biomed. Res. Int. 2013, 2013, 349129. [CrossRef]

52. Berger, L.M.; Blank, R.; Zorn, F.; Wein, S.; Metges, C.C.; Wolffram, S. Ruminal degradation of quercetin and its influence on fermentation in ruminants. J. Dairy Sci. 2015, 98, 5688-5698. [CrossRef]

53. Stoldt, A.-K.; Derno, M.; Das, G.; Weitzel, J.M.; Wolffram, S.; Metges, C.C. Effects of rutin and buckwheat seeds on energy metabolism and methane production in dairy cows. J. Dairy Sci. 2016, 99, 2161-2168. [CrossRef]

54. Guglielmelli, A.; Calabro, S.; Primi, R.; Carone, F.; Cutrignelli, M.I.; Tudisco, R.; Piccolo, G.; Ronchi, B.; Danieli, P.P. In vitro fermentation patterns and methane production of sainfoin (Onobrychis viciifolia Scop.) hay with different condensed tannin contents. Grass Forage Sci. 2011, 66, 488-500. [CrossRef]

55. Rodríguez Hernáez, J.; Cerón Cucchi, M.E.; Cravero, S.; Martinez, M.C.; Gonzalez, S.; Puebla, A.; Dopazo, J.; Farber, M.; Paniego, N.; Rivarola, M. The first complete genomic structure of Butyrivibrio fibrisolvens and its chromid. Microb. Genom. 2018, 4, e000216. [CrossRef] [PubMed]

56. Broudiscou, L.-P.; Lassalas, B. Effects of Lavandula officinalis and Equisetum arvense dry extracts and isoquercitrin on the fermentation of diets varying in forage contents by rumen microorganisms in batch culture. Reprod. Nutr. Dev. 2000, 40, 431-440. [CrossRef] [PubMed]

57. Bodas, R.; López, S.; Fernández, M.; García-González, R.; Rodríguez, A.B.; Wallace, R.J.; González, J.S. In vitro screening of the potential of numerous plant species as antimethanogenic feed additives for ruminants. Anim. Feed Sci. Technol. 2008, 145, 245-258. [CrossRef]

58. Patra, A.; Park, T.; Kim, M.; Yu, Z. Rumen methanogens and mitigation of methane emission by anti-methanogenic compounds and substances. J. Anim. Sci. Biotechnol. 2017, 8, 13. [CrossRef] [PubMed]

59. Saminathan, M.; Gan, H.M.; Abdullah, N.; Wong, C.M.V.L.; Ramiah, S.K.; Tan, H.Y.; Sieo, C.C.; Ho, Y.W. Changes in rumen protozoal community by condensed tannin fractions of different molecular weights from a Leucaena leucocephala hybrid in vitro. J. Appl. Microbiol. 2017, 123, 41-53. [CrossRef] [PubMed]

60. Salami, S.A.; Valenti, B.; Bella, M.; O’Grady, M.N.; Luciano, G.; Kerry, J.P.; Jones, E.; Priolo, A.; Newbold, C.J. Characterisation of the ruminal fermentation and microbiome in lambs supplemented with hydrolysable and condensed tannins. FEMS Microbiol. Ecol. 2018, 94, 61. [CrossRef]

61. Montoya-Flores, M.D.; Molina-Botero, I.C.; Arango, J.; Romano-Muñoz, J.L.; Solorio-Sánchez, F.J.; Aguilar-Pérez, C.F.; Ku-Vera, J.C. Effect of dried leaves of Leucaena leucocephala on rumen fermentation, rumen microbial population, and enteric methane production in crossbred heifers. Animals 2020, 10, 300. [CrossRef]

62. MacAdam, J.W.; Villalba, J.J. Beneficial effects of temperate forage legumes that contain condensed tannins. Agriculture 2015, 5, 475-491. [CrossRef]

63. Komáromyová, M.; Mravčáková, D.; Petrič, D.; Kucková, K.; Babják, M.; Dolinská, M.U.; Königová, A.; Mad’arová, M.; Pruszyńska-Oszmałek, E.; Cieslak, A.; et al. Effects of medicinal plants and organic selenium against ovine haemonchosis. Animals 2021, 11, 1319. [CrossRef]

64. Cériac, S.; Jayles, C.; Arquet, R.; Feuillet, D.; Félicité, Y.; Archimède, H.; Bambou, J.C. The nutritional status affects the complete blood count of goats experimentally infected with Haemonchus contortus. BMC Vet. Res. 2017, 13, 326. [CrossRef] [PubMed]

65. Torres-Acosta, J.F.J.; Jacobs, D.E.; Aguilar-Caballero, A.; Sandoval-Castro, C.; Cob-Galera, L.; May-Martínez, M. Improving resilience against natural gastrointestinal nematode infections in browsing kids during the dry season in tropical Mexico. Vet. Parasitol. 2006, 135, 163-173. [CrossRef] [PubMed]

66. Eberle, J.U.; Voehringer, D. Role of basophils in protective immunity to parasitic infections. Semin. Immunopathol. 2016, 38, 605-613. [CrossRef] [PubMed]

67. Lins, J.G.G.; Almeida, F.A.; Amarante, A.F.T. Haematological variables of Santa Ines and Ile De France suckling lambs: Influence of Haemonchus contortus infection. Pesq. Vet. Bras. 2020, 40, 882-891. [CrossRef] 
68. Stear, M.J.; Bishop, S.C.; Henderson, N.G.; Scott, I. A key mechanism of pathogenesis in sheep infected with the nematode Teladorsagia circumcincta. Anim. Health Res. Rev. 2003, 4, 45-52. [CrossRef]

69. Blanchard, J.L.; Gallina, A.M.; Wescott, R.B. Pathologic changes in lambs with Ostertagia circumcincta infections associated with decreased infectivity of Haemonchus contortus. Am. J. Vet. Res. 1986, 47, 309-314.

70. Mravčáková, D.; Sobczak-Filipiak, M.; Váradyová, Z.; Kucková, K.; Čobanová, K.; Maršík, P.; Tauchen, J.; Vadlejch, J.; Mickiewicz, M.; Kaba, J.; et al. Effect of Artemisia absinthium and Malva sylvestris on antioxidant parameters and abomasal histopathology in lambs experimentally infected with Haemonchus contortus. Animals 2021, 11, 462. [CrossRef] 